\title{
Experiencias en la atención médica de madres de niños y niñas con síndrome de Down. Estudio cualitativo con técnicas etnográficas
}

Alejandra Lopera-Escobar, ${ }^{1}$ Myriam Bastidas-Acevedo ${ }^{2}$

\section{Resumen}

INTRODUCCIÓN: los significados de la discapacidad que se construyen en la interacción entre el médico y las familias de niños con síndrome de Down influyen en el desarrollo durante la primera infancia y reflejan las actitudes sociales que experimentan las personas con discapacidad y sus familias, específicamente en contextos como la atención médica.

OBJETIVO: describir las experiencias en la atención médica de las madres de niños y niñas con síndrome de Down y su influencia en la construcción del significado de esta condición.

MATERIAL Y MÉTODO: estudio cualitativo con técnicas etnográficas efectuado en Medellín, Colombia, en 2016. Se entrevistó a madres de niños con síndrome de Down, menores de 6 años, previo consentimiento informado. Se efectuó análisis simultáneo de la información y revisión concomitante de la bibliografía con la emergencia del dato.

RESULTADOS: se entrevistaron 17 madres que manifestaron insatisfacción con la atención médica de sus hijos por la falta de reconocimiento del niño con síndrome de Down, por la negación del derecho a la salud y la deshumanización de la atención.

CONCLUSIONES: las madres experimentaron una relación de poder asimétrica en la consulta médica. La posición dominante del profesional se impone a la madre, a los derechos y necesidades del niño con SD. Las madres cuestionaron la praxis y el entrenamiento médico.

PALABRAS CLAVE: atención médica, síndrome de Down, derechos, discapacidad.
${ }^{1}$ Fisioterapeuta, Magister en Salud Pública.

${ }^{2}$ Médica Pediatra Magister en Salud Colectiva. Docente.

Universidad de Antioquia, Colombia.

Recibido: 29 de enero 2017

Aceptado: 28 de julio 2017

Correspondencia

Alejandra Lopera Escobar alejaloperae@gmail.com

Este artículo debe citarse como Lopera-Escobar A, Bastidas-Acevedo M. Experiencias en la atención médica de madres de niños y niñas con síndrome de Down. Estudio cualitativo con técnicas etnográficas. Acta Pediatr Mex. 2018;39(2):109-120. 


\section{Experiences of mothers of children with Down syndrome in medical care. Qualitative study with ethnographic techniques}

Alejandra Lopera-Escobar, ${ }^{1}$ Myriam Bastidas-Acevedo ${ }^{2}$

\begin{abstract}
INTRODUCTION: The meanings of disability that are built in the interaction between the physician and the families of children with Down Syndrome (DS) influence childhood development and reflect the attitudes experienced by people with disabilities and their families in society, specifically In contexts such as medical care.
\end{abstract}

OBJECTIVE: To describe the experiences of mothers of children with DS in the medical consultation of their children and its influence in the construction of the meaning of DS

MATERIAL AND METHOD: Qualitative study with ethnographic techniques carried out in Medellin-Colombia in 2016; we interviewed 17 mothers of children with DS. Simultaneous analysis of the information and concomitant review of the literature were performed with the emergence of the data.

FINDINGS: Mothers expressed dissatisfaction with their children's medical care; for the lack of recognition of the child with DS, for the denial of the right to health and the dehumanization of care.

CONCLUSIONS: The dominant position of the professional was imposed on the mother, the rights and needs of the child with DS. Mothers questioned praxis and medical training; emerged the lack of recognition of the child with DS and his mother, the denial of the right to health and the dehumanization of medical care.

KEYWORDS: medical care; Down syndrome; rights; disability

\author{
${ }^{1}$ Fisioterapeuta, Magister en Salud Pública. \\ ${ }^{2}$ Médica Pediatra Magister en Salud Colec- \\ tiva. Docente. \\ Universidad de Antioquia. \\ Correspondence \\ Alejandra Lopera Escobar \\ alejaloperae@gmail.com
}

\section{INTRODUCCIÓN}

Un nuevo enfoque del desarrollo humano ha transformado la manera en que asumimos las libertades del hombre, sus límites, medios y fines. ${ }^{1}$ La visión de discapacidad se introduce en este enfoque partiendo del reconocimiento de la diversidad de funcionamientos y oportunidades dispuestas al alcance de las personas para vivir dignamente. ${ }^{2}$ Desde esta perspectiva, la disca- pacidad es un fenómeno social, resultado de la interacción entre la identidad de un sujeto y sus respectivos funcionamientos en su contexto ambiental y actitudinal. ${ }^{3}$ Dicho esto, la atención médica es uno de los ámbitos contextuales en los que se manifiesta la discapacidad, se comunican asuntos biológicos, diagnósticos, pronósticos y se disponen oportunidades para mantener y recuperar la salud como un recurso indispensable para la vida. 
El espacio y tiempo dispuesto para que el médico se aproxime a la realidad biológica de un sujeto, su estado de salud y enfermedad, se presta para la observación mutua, interacción, intercambio de preguntas, comunicación de ideas, sentidos y emociones, contacto físico, incluso renuncia de sí mismo para confiar la vida en el otro.

El tacto, el tono de voz, las palabras, además de la mirada del otro y su juicio de valor acerca del estado de salud de una persona, siembran conceptos sobre sí, que modelarán comportamientos concernientes a la preservación de la vida. Hace más de 2000 años, para Hipócrates, el médico debía reunir entre sus habilidades más importantes: sabiduría, humanidad e integridad; para Pedro Laín Entralgo, es indispensable en el médico, la empatía, como el saber ponerse en el lugar del otro y disponerse en su ayuda. ${ }^{4}$

Dado que las personas construyen significados de acuerdo con sus experiencias y formación, la mirada que el profesional de la salud tiene de los sujetos beneficiarios de su conocimiento disciplinar, en este caso, niños con discapacidad, está fundada en sus experiencias de vida, su propia cultura de crianza y el conocimiento desarrollado durante su formación académica. Por tanto, hay un sistema de significados que amerita ser reconocido y comprendido en la atención médica. ${ }^{5}$

El síndrome de Down (SD), además de reconocerse como una discapacidad en un individuo, es una forma de vida con funcionamientos particulares, que según las oportunidades, podrá desarrollar sus capacidades para la inclusión social a la que tiene derecho. El exceso de material genético correspondiente a un cromosoma 21 adicional, genera repercusiones biológicas y psicológicas que al interactuar con el medio social trascienden en la calidad de vida del niño, su familia y la posibilidad de incluirse socialmente. ${ }^{6}$
Estudios en Latinoamérica evidencian que los prejuicios sobre la discapacidad influyen en la calidad de la atención en salud que reciben los niños con síndrome de Down, ${ }^{7,8}$ evidenciando la inequidad y las barreras que deben superar estos niños y sus familias para recibir la atención que necesitan.

La familia en el proceso de crianza y desarrollo del niño con síndrome de Down, por ser el entorno directo y primario del mismo, requiere acompañamiento que fomente la conciencia acerca de las capacidades, las potencialidades a desarrollar y aceptar la discapacidad. Los médicos envestidos con la legitimidad dada desde las instituciones formadoras de recurso humano tienen una gran influencia en las creencias que las madres se forman acerca de sus hijos con síndrome de Down.

Si los niños y niñas con síndrome de Down no reciben el amor, el estímulo sensorial, la atención sanitaria y la inclusión social a la que tienen derecho, puede desaprovecharse el periodo crítico del desarrollo, lo que limitará injustamente sus posibilidades y tendrán importantes implicaciones económicas y sociales para ellos mismos, sus familias y las comunidades de las que hacen parte. ${ }^{9}$

Por ello, comprender las experiencias de las madres, como protagonistas de la crianza, permite reconocer aspectos que influyen en el desarrollo durante la primera infancia de los niños con síndrome de Down y reflejan las actitudes que experimentan las personas con discapacidad y sus familias en la sociedad, específicamente en contextos como la atención médica.

\section{OBJETIVOS}

Describir las experiencias de las madres de niños con síndrome de Down, menores de seis años, en la atención médica de sus hijos en la ciudad de Medellín, Colombia. 
Describir la influencia de la atención médica en la construcción del significado del síndrome de Down en las madres de pacientes menores de seis años.

\section{MATERIAL Y MÉTODO}

Investigación cualitativa con técnicas etnográficas en la que se invitó a participar a madres de niños con síndrome de Down menores de seis años, que asistían a los programas de salud y rehabilitación integral de dos instituciones de la ciudad de Medellín, Colombia.

Se decidió escuchar la voz de las madres dado que en investigaciones, tanto nacionales como internacionales, se encontró que las madres son las cuidadoras principales de los niños; se reconoce que la experiencia de cuidadores como padres, abuelas y otros es importante, pero no fueron sujetos de esta investigación. Por tanto, los criterios de inclusión de este estudio fueron: ser madre de un niño o niña menor de 6 años con síndrome de Down, manifestar su deseo voluntario de participar en la investigación y firmar el consentimiento informado.

La recolección de la información se hizo mediante entrevistas en profundidad, cara a cara entre la investigadora y las informantes, en un espacio facilitado por una de las instituciones vinculadas al estudio. Es de anotar que no había una relación previa entre la investigadora principal y las informantes; sin embargo, la interacción a lo largo de la investigación evolucionó generando un ambiente de confianza para relatar las experiencias. Las entrevistas fueron orientadas por preguntas abiertas y tuvieron una duración de aproximadamente 45 minutos cada una. ${ }^{10}$ Con el consentimiento de las madres se grabó el audio de cada una de las entrevistas para su posterior transcripción y análisis. Se inició con muestreo en bola de nieve que evolucionó a un muestreo teórico, en el que la saturación de los datos marcó el límite de la muestra, el cual fue alcanzado con 17 participantes en 21 entrevistas, debido a que con dos informantes se realizaron dos encuentros para profundizar los tópicos emergentes.

El análisis se hizo simultáneo a la recolección de la información, con un patrón cíclico de trabajo para ordenar, reducir e interpretar los datos recogidos, lo que permitió reformular y evolucionar las entrevistas posteriores. Se realizó codificación abierta y posteriormente axial, consignando los códigos y categorías en un archivo del programa Excel $^{\circledR}$ de Office. Con el fin de absorber e identificar patrones de experiencia, en el análisis tuvo gran importancia la información que se repitió en diferentes entrevistas, así como los datos excepcionales.

Al final del estudio las madres participaron en un taller de investigación con el propósito de hacer la triangulación y validación de las categorías que emergieron en el análisis. ${ }^{11}$ La calidad del proceso de investigación se contrastó con la luz de tres criterios de validez: credibilidad, auditabilidad y transferibilidad. ${ }^{12}$

\section{RESULTADOS}

Las participantes tenían entre 21 y 52 años; afiliadas al Sistema General de Seguridad Social en Salud, 10 de ellas al régimen contributivo y 7 al régimen subsidiado (existen dos regímenes de aseguramiento en salud en Colombia; el Régimen Contributivo al que deben afiliarse las personas vinculadas a través de contrato de trabajo y los trabajadores independientes con capacidad de pago y el Régimen Subsidiado en el que están afiliadas las personas sin capacidad de pago). En cuanto a la escolaridad, seis madres tenían básica primaria, nueve madres educación secundaria y dos madres con educación técnica; en relación con la ocupación, quince de las madres eran amas de casa y dos tenían trabajos informales.

Se efectuó análisis simultáneo de la información y revisión concomitante de la bibliografía con la 
emergencia del dato. Se hallaron 680 códigos descriptivos que se agruparon en 8 categorías, que a su vez conformaron dos supra categorías: el reconocimiento del niño y la madre y la relación de poder en la atención médica.

\section{El reconocimiento del niño y la madre en la atención médica}

Esta categoría hace referencia a lo que las madres expresaron en relación con el respeto en la atención médica, la valoración del niño, el reconocimiento del niño como sujeto de derechos y de su singularidad, además de la consideración que tuvieron con ellas en la atención médica.

\subsection{Experiencias dignificantes}

En la atención médica emergieron unas experiencias excepcionales que, aunque poco frecuentes, demuestran que sí es posible una atención empática y humanizada. La confianza fue un aspecto relevante para las madres, ya que les permitió sentirse cómodas preguntando y solicitando orientación, de esta manera obtuvieron de la atención y del profesional lo que buscaban y necesitaban para sus hijos. Las madres valoraron el buen trato al niño en la atención médica, que lo miren y que le hablen, que el niño sea el protagonista y el centro de atención del médico.

"Hay una médica, que me encanta porque lo trata muy bien y porque lo recibe muy bien y porque el saludo es maravilloso... cuando voy a consulta, le va hablando al niño: $i . . . y ~ q u e$ estás haciendo? ¿Cómo se llama tu profe? ¿Estás estudiando? O sea, ella me va hablando a mí, me va preguntando, pero con el niño va por los laditos... Me encanta, me encanta el carisma de esa doctora". (E10 p. 8).

\subsection{Experiencias no dignificantes}

Las madres mencionaron la importancia del trato que tuvo el médico hacia el niño o niña con síndrome de Down, algunos médicos fueron cá- lidos, saludaban, interactuaban con el niño; pero en otros casos el niño era invisible en la atención, no lo miraban, no lo reconocían como sujeto.

"Hay unos médicos que son muy amables, hay otros que no se dirigen a la niña si no a mí y a la niña me la aíslan sabiendo que si es ella la que viene a consulta ella es la paciente, en el momento deberían decirle: ¿qué te duele? Entonces son: mamá dígale que abra la boca, mamá dígale que tal cosa..." (E3 p. 4).

El trato hacia el niño es percibido de manera personal por la madre, es decir, si el médico reconoce al niño con síndrome de Down, la madre se siente reconocida, si el niño es ignorado, la madre se siente ignorada e incómoda. Algunas percibieron incluso agresividad y palabras ofensivas.

"La pediatra nos dijo si veíamos que la niña era normal, o sea llegó siempre como un poquito agresiva y dijo que si la veíamos normal; nos preguntó al papá y a mí y entonces nosotros dijimos: si y ella nos miró y nos dijo: ¡no! ella no es normal". (E4 p. 1.)

\subsubsection{Significado del niño con síndrome de Down en la atención médica}

Desde las experiencias de las madres, en la interacción con el médico surgen comentarios y apreciaciones acerca del niño con síndrome de Down, de su condición y sus características. Dichas apreciaciones se inclinan por resaltar rasgos y atributos que no están presentes en niños sin esta condición y que consideran que no son normales. En la atención, los niños con síndrome de Down pierden su identidad y, por tanto, su condición los define. Las madres escucharon adjetivos ofensivos y discriminantes que se oponen a lo que ellas piensan de sus hijos:

"... Entonces ya un doctor pues muy bruscamente dijo: no, ¿ustedes no conocen los mongolos? Él es un mongolo". (E11 p. 8). 
"Yo le dije al doctor, usted qué me dice en cuanto al cariotipo, y me dijo: no, eso es cuestión de mala suerte, de 100 le tocó a usted, eso es cuestión de mala suerte." (E1 p. 6).

"Yo creo que los pediatras ven a Mateo como que no va a ser nada, ni nadie, pues no le va a servir a la sociedad; pues así: ay, otro ahí del montón, otro pa' que esté en la casa". (E8 p. 10).

\subsubsection{Negación del derecho a la salud}

La negación de los derechos en salud del niño, con relación a la omisión del seguimiento a las comorbilidades del síndrome de Down, remisión a especialistas y a pruebas diagnósticas, es un asunto frecuente en las consultas médicas ya sea por desconocimiento del médico, por restricciones administrativas de su sitio de trabajo o empleador (IPS), por impericia o negligencia.

"...yo le dije a los médicos: ¿ustedes por qué no me le hacen un cariotipo a la niña? Y me dijeron: no te lo podemos hacer. ¿Por qué? Porque es de alto costo y la EPS no te va a cubrir eso". (E3 p. 2).

En cuanto a pruebas diagnósticas, las madres se enteran, por otras madres de niños con síndrome de Down u otros profesionales, que los niños requieren exámenes de forma regular, como los potenciales evocados visuales y auditivos, rayos $X$ de cadera, controles por cardiología, entre otros. Cuando la madre solicita dichos exámenes al médico, el profesional responde que el niño no necesita el examen o que no puede prescribirlo.

"La doctora dijo: ¿y usted para qué había venido? Y yo: porque el niño necesita los exámenes de los ojos y los oídos. Y la pediatra empezaba a aplaudirle (palmadas) no, él está bien, y lo puso a seguir un lápiz, no, él sigue el lápiz ¿para qué? $Y$ yo: no, pero es que lo necesita por si algo, uno no sabe; y ella dijo: no, él está bien, entonces no le mandó los exámenes". (E8 p. 6).
En cuanto a las terapias de habilitación integral, a las que tienen derecho todas las personas con discapacidad y cuyo propósito establecido por la ley estatutaria 1618 de 2013 de Colombia, es el "mejoramiento de la calidad de vida y la plena integración de la persona con discapacidad al medio familiar, social y ocupacional, a través de procesos terapéuticos, educativos y formativos que se brindan acorde al tipo de discapacidad"13 es en muchos casos vulnerado. Prevalece en nuestra sociedad un desconocimiento de este derecho que se evidencia tanto en la atención médica como en las personas con discapacidad y sus familias, asunto que en el caso de los niños con SD influye en las oportunidades y sus posibilidades de desarrollarse e incluirse en la sociedad.

"El fisiatra sentado allá en el computador: a ver ¿qué tiene?; le dije yo: el niño necesita las terapias, y dijo: ¿él qué terapias va a necesitar? ...Y yo: ¿Cómo que no las necesita? Si él está avanzado es porque está en terapias, me dijo: vea mamá él está bien, tiene fuerza, ¿qué más quiere?". (E9 p. 5).

Desde la experiencia de las madres, los médicos asumían una actitud en la que subyace un desconocimiento de la necesidad de las terapias de habilitación integral, desconociendo que el niño está en el periodo crítico del desarrollo y que dada su condición requería de diferentes tipos de terapias para estimular todas sus áreas del desarrollo y así mejorar su calidad de vida. Este comportamiento representa una vulneración de derechos que desde otro punto de vista era una forma de ahorrar recursos en salud, sin importar el costo que esto constituye para la vida misma del niño.

"Con el neurólogo me fue muy mal porque yo necesitaba que me diera la orden para las terapias y me dijo que eso ya no lo mandaban, que esos niños ya no necesitan eso". (E12 p. 11). 


\subsubsection{La madre frente a los derechos del niño}

Las madres refirieron que desconocían los derechos en salud de sus hijos, algunas se enteraron del derecho y necesidad que tienen los niños de recibir rehabilitación integral al interactuar con otras madres de niños con síndrome de Down o buscando información por otros medios. En estos casos, el médico de familia tratante y el pediatra no lo remitieron a rehabilitación integral ni a otro tipo de terapias. Por tanto, las madres se remiten a instancias legales para reclamar los derechos en salud de sus hijos.

"...la niña nació y sí me le mandaron los exámenes, pero ya. Nunca me dijeron que había que hacerle terapias, que había que... nada". (E6 p. 1).

"Me siento como ignorada, como que no soy valorada, como que la niña... no me la insulta, no me tratan mal, pero con el hecho de no darme lo que la niña necesita yo con eso ya quedo demasiado, como afligida". (E4 p. 5).

Los trámites y diligencias que deben asumir las madres para lograr que sus hijos reciban los servicios de salud a los que tienen derecho, evidencian las barreras que las personas con discapacidad y sus familias deben superar en el contexto del Sistema de Seguridad Social en Colombia.

\section{La relación de poder en la atención médica}

La relación que se establecía entre el médico y la madre en la consulta del niño con síndrome de Down, encarnaba una interacción social cargada de símbolos, fundados a partir de la formación académica, de los conocimientos, de los significados acerca del niño con síndrome de Down, de las experiencias y de la cultura misma de cada uno de ellos.

Referente a esta relación se encontraron asuntos de orden discursivo y de orden práctico que, según las madres, influyeron en la construcción subjetiva del significado del síndrome de Down y en la atención en salud que recibieron los niños.

\subsection{La atención médica centrada en el déficit}

Según los relatos de las madres de este estudio se encontró que, en la atención médica de niños con síndrome de Down, el protagonista o foco de la atención fue el déficit, es decir, lo que se dijo durante la atención médica tiene un marcado enfoque morbicéntrico, que redujo al individuo a su dimensión biológica. El discurso del médico se basaba, según las experiencias de las madres, en la enumeración de los "problemas", limitaciones y riesgos propios del síndrome de Down, no se hizo mención de las posibilidades, las capacidades y potencialidades de los niños.

"El pediatra me explicó que nacían con muchos problemas de salud, del corazón, de los ojos, de los oídos... que eran unos niños que no iban a ir a la escuela, no iban a ver una guardería, en resumidas cuentas, que no iban a servir para nada." (E7 p. 1).

\subsection{La desesperanza}

"No hay nada por hacer" fue el mensaje desesperanzador que muchas de las madres escucharon durante la atención médica. La experiencia del médico y sus conocimientos se enfocaron en las condiciones asociadas a la trisomía 21 y a las complicaciones que pueden presentarse, con una mirada reducida del individuo que omitió las capacidades, las subjetividades y la diversidad.

"... a veces le dicen a uno que porque el niño tiene un Down, como que no hay nada que hacerle, que uno lo deje así, pero a mí no me parece porque mi niño tiene mucho para dar". (E5 p. 7).

La formación del médico centrada en lo biológico, sumada a las creencias sociales frente 
a la discapacidad e incluso la crianza que los profesionales vivieron en sus familias se asoció a este discurso desesperanzador escuchado por algunas de las madres de esta investigación.

"Todos los días el doctor me recordaba que la niña tenía síndrome de Down, entonces si la niña estaba muy congestionada, decía: mamá es que ella va a durar muy congestionada porque tiene síndrome de Down. Doctor ¿cómo va el progreso de la niña? me decía: no, la niña se demora porque como ella tiene síndrome de Down". (E17 p. 3).

\subsection{La culpa}

Las madres expresaron que algunos médicos las culpabilizaron por haber tenido un hijo con síndrome de Down, las responsabilizaron de la condición del niño, se les cuestionó y señaló por tener hijos a edad avanzada. Estos comentarios que tuvieron lugar en la consulta generaron en las madres culpa por las dificultades, las enfermedades de su hijo y las barreras que deberán superar en el futuro, que pudieron ser evitadas de no haber tenido hijos a su edad. Fueron pensamientos recurrentes que han generado depresión y tristeza en las madres.

"Me sentía muy mal, yo no sabía ni que decirle porque a pesar que ya nosotros sabíamos que la edad sí influía, pero que pudo haber sido por otras razones. Yo a lo último le dije: yo con usted no voy a pelear, usted gana. Prácticamente ella ganó". (E7 p. 9).

\subsection{La omisión en la atención médica}

Las madres en su experiencia de crianza aprendieron que sus hijos necesitaban valoraciones diferenciales. Sin embargo, en la atención médica percibieron desinterés por el desarrollo integral del niño, identificaron omisiones, pocos médicos preguntaron si el niño iba al jardín, a alguna institución educativa, o si realizaba de forma independiente las actividades básicas cotidianas tales como la alimentación, el vestido, el aseo, entre otras, que dan cuenta del desarrollo psicomotor del niño. Las madres mencionaron reiteradamente que el profesional en la consulta no se interesó por el contexto del niño, no reconoció sus necesidades particulares, ni las de la familia.

“...con algunos pediatras era muy incómodo y por eso pasamos por tantos, porque a él no le prestaban atención. Uno veía que no lo atendían según la condición que él tiene, porque uno sabe que en la condición en la que ellos nacen necesitan ciertos cuidados o más cuidados". (E8 p. 3).

Las madres de esta investigación consideraron que la consulta, incluso desde la etapa prenatal, no llenó sus expectativas ni las necesidades del niño, dado que no se hicieron diagnósticos oportunos, la atención no fue diferencial y los equipararon a niños sin el síndrome. Por lo tanto, salieron del encuentro con el profesional angustiadas y con muchos asuntos sin resolver.

"...siempre las consultas son como muy pobres, que uno sale como en las mismas y uno dice ¿yo a qué vine? simplemente como por cumplir, bueno yo como mamá, por cumplir". (E4 p. 4).

"yo salí de allá de la consulta con el niño y yo dije: no pues, a nada vine... a nada vine". (E9 p. 6).

Aunque todas las madres entrevistadas tuvieron controles prenatales y ecografías periódicas, solo una de ellas tuvo diagnóstico prenatal del síndrome de Down, otras fueron informadas inmediatamente después del parto y una incluso se enteró cuando su hija ya tenía 3 meses de vida.

"El especialista nunca me dijo: mamá la niña viene con problemas, nunca. De hecho las ecografías que me hicieron me decían que venía pequeña pero sin ningún problema". (E3 p. 1). 
La consulta médica de niños con síndrome de Down según las madres no fue diferencial, se omitieron aspectos importantes para la salud del niño, no se tuvieron en cuenta las necesidades particulares asociadas a la condición y no se realizó el seguimiento de comorbilidades.

\section{Propuestas de las madres para la consulta médica}

Las madres en el proceso de recordación de las experiencias que tuvieron en la atención médica de sus hijos, verbalizaron lo que esperaban del profesional médico; señalaron la importancia de que sus hijos fueran vistos como niños y no reducidos a su condición de síndrome de Down. Propusieron una atención humanizada, en la que fueran reconocidas, escuchadas, respetadas y comprendidas. Esperan un médico empático que se interese por el bienestar del niño, que las oriente y las ayude.

“...yo creo que a los médicos les hace falta ser más humanos, ponerse en el lugar de uno, de la angustia en la que uno llega a pedir algo, a buscar una ayuda, no una solución, pero si una ayuda de cualquier problema de salud". (E1 p. 11).

\section{DISCUSIÓN}

La atención médica de los niños con síndrome de Down: una relación de poder

Uno de los resultados más representativos de este estudio, y como eje de significación en el análisis, fue la relación que experimentaron las madres durante la consulta, la cual, en experiencias excepcionales, fue una relación de confianza que se manifiesta en la comunicación horizontal entre la madre y el profesional en la consulta; sin embargo, en la mayoría de las experiencias relatadas se identificó una relación de poder asimétrica, percibida por las madres como una fuerza dominante asumida por el médico desde la forma como se comunicó, relacionó y practicó su conocimiento técnico en el espacio concreto de la atención médica.

En la mayoría de las experiencias de esta investigación se evidenció una relación vertical entre el profesional, la madre y el niño con síndrome de Down. El médico doblemente legitimado por su formación académica y por la institución a la que pertenece ${ }^{14}$ expresó una actitud y un discurso irrefutable que predominó en la atención médica. Según Lolas, "los discursos poseen poder: indican, comandan, prescriben, proscriben, prohíben, permiten"15 y es justo este poder que experimentaron las madres cuando el profesional emitió un diagnóstico, pronóstico y determinó una conducta relacionada con la salud del niño con síndrome de Down; se desconoció su saber empírico, ${ }^{16}$ en muchas ocasiones se contradijeron y rechazaron sus solicitudes.

Para comprender la relación de poder que se da en la atención médica donde se comunican aspectos ligados a la salud, se retoma a Foucault quien afirma que: "Las relaciones de comunicación producen efectos de poder por el solo efecto de que modifican el campo informativo de quienes participan en ella"17 lo cual implica que el poder que ejerció el médico en la atención también estuvo asociado a su capacidad de influir en los conocimientos y creencias de la madre con la información que le transmitió acerca del niño con síndrome de Down; sin embargo, las madres lograron aprendizajes desde su experiencia de crianza que les permitió transformar esas creencias, reconocer al niño con síndrome de Down como un sujeto, con capacidades y derechos ${ }^{18}$ y por tanto resignificar la información que recibieron del médico.

La visión reduccionista y netamente biológica que se expresó en la atención médica está asociada a la formación médica actual, que reproduce un modelo médico hegemónico, fundado por el pensamiento occidental y el positivismo, cuya racionalidad mecanicista reduce al 
individuo a su dimensión física, desconociendo las dimensiones social, psíquica, emocional y espiritual. ${ }^{19,20}$ Así mismo y retomando a Foucault con la dicotomía normal y anormal en "Los Anormales", ${ }^{21}$ el síndrome de Down representa para los médicos una enfermedad o desviación que debe ser intervenida, medicada y normalizada.

Enmascarado en el discurso morbicéntrico, las madres percibieron rechazo a la diferencia y un señalamiento de que el niño no se desarrolla como los demás, que el síndrome de Down es una condición sin cura, es para toda la vida y por tanto no aportará a la sociedad como las personas sin esta condición. Estas creencias se reprodujeron en el espacio de la atención médica en forma de discriminación, señalamiento y negación del derecho a la salud.

\section{El reconocimiento del niño con síndrome de Down y su madre en la atención médica}

Para la comprensión de las experiencias de las madres en la atención médica de sus hijos, se retoma la teoría del Reconocimiento de Axel Honneth, con la premisa de que los individuos solo se constituyen como seres humanos en relación con otros, en un espacio intersubjetivo de interacción; ${ }^{22}$ es decir, que se necesita del otro y de la relación que se establece con él para entenderse en la sociedad. ${ }^{23}$

La interacción entre el médico, el niño con síndrome de Down y la madre desde las experiencias de esta última arrojó aspectos relacionados con el proceso intersubjetivo del reconocimiento, la falta de éste y sus repercusiones morales planteados por Honneth. ${ }^{24}$

En esta investigación, aunque fueron muy pocas las experiencias positivas en la atención médica, resaltaron aquellas en las que el niño recibió un trato cálido y amable, fue visibilizado como sujeto protagonista de la atención y fue visto desde las capacidades. Estas experiencias re- presentaron el reconocimiento y dignificación que la madre y el niño necesitan en su proceso intersubjetivo de significación social.

Los hallazgos del presente estudio también revelaron la falta de reconocimiento del niño con síndrome de Down y de su madre, asuntos que además de generar heridas morales en ellos, sustentan las desventajas que tienen en la sociedad en términos de buen trato, de oportunidades y goce efectivo de derechos.

En la teoría de Honneth, el reconocimiento se constituye en tres esferas: el amor, entendida en un sentido amplio del cuidado; la esfera del derecho que busca la autonomía de los sujetos haciendo posible el libre ejercicio de sus capacidades; y la esfera de la solidaridad que hace referencia a la valoración social de las características particulares de los individuos. ${ }^{22}$ La trasgresión de cada esfera se traduce en una herida moral específica para el individuo. En este estudio se encontró que las madres en la esfera del amor experimentaron maltrato; en la esfera del derecho, hubo negación y discriminación; y finalmente en la esfera de la solidaridad, el daño fue la estigmatización de la que fueron objeto los niños con síndrome de Down. Estos daños infringen la autoconfianza, el autorrespeto y la autoestima elementos constitutivos para el ser humano y para su interacción en la sociedad.

La trasgresión en la esfera del derecho que en este estudio se evidenció, en la negación del derecho a la salud del niño con síndrome de Down, representó el no reconocimiento de la capacidad moral del niño y el no reconocimiento como sujeto autónomo digno de derechos y deberes.

En relación con el significado del síndrome de Down que se reproduce en la atención médica, las madres experimentaron un tipo de herida moral, que, manifestada como humillación y falta de respeto, trasmitió el mensaje de que sus facultades e incluso las de sus hijos no merecían 
reconocimiento alguno. Este tipo de herida moral representó una frustración en esa lucha humana por tener significación social en una comunidad. ${ }^{24}$

\section{La negación del derecho a la salud}

El derecho a la salud en su expresión más reducida, que corresponde al acceso y prestación del servicio de salud, fue precisamente el derecho que se vulneró a los niños con síndrome de Down según las experiencias de las madres en la atención médica. Obviando el derecho a la protección social, a la educación de calidad e incluyente, a la vivienda, a una nutrición adecuada, a la inclusión social, entre otros derechos constitutivos para el derecho a la salud y la dignidad humana en su expresión más amplia. ${ }^{25}$ Este estudio refleja que la negación fue una constante en el contexto de la prestación de servicios que involucra asuntos administrativos, actitudes e imaginarios acerca de la discapacidad y demuestra el poder que tiene el profesional en la atención médica para dictaminar que aprobar o negar en servicios de salud yendo en contra de las necesidades del paciente, incluso de las guías de práctica clínica internacionalmente avaladas.

La brecha existente entre la legalidad y la legitimidad de los derechos resalta en los hallazgos de esta investigación, en algunos casos por desconocimiento, la mayoría de los casos por la falta de reconocimiento del niño con síndrome de Down como sujeto de derechos y en otros, por la racionalidad instrumental que prevalece en nuestra sociedad.

La premisa de que las personas con discapacidad no son productivas para una sociedad capitalista ${ }^{26}$ y que los recursos invertidos en esta población no serán recuperados en términos de productividad y fuerza de trabajo, está impresa culturalmente en la atención médica según las experiencias de este estudio. La reproducción de este pensamiento instrumental, consciente o inconscientemente por parte de los profesionales, se traduce en la negación y el ahorro de recursos en salud. Las terapias de habilitación y otros apoyos terapéuticos, desde esta perspectiva son un gasto injustificado que no logrará que el niño con síndrome de Down viva y se desarrolle como una persona sin esta condición.

\section{CONCLUSIÓN}

Algunas madres de este estudio experimentaron en la atención médica de sus hijos una relación de confianza con el profesional; sin embargo, la mayoría experimentaron una relación de poder asimétrica, en la cual la postura dominante del saber científico del profesional se impuso al conocimiento práctico de la madre, a los derechos y a las necesidades que tiene el niño con síndrome de Down.

En la mayoría de las experiencias en la atención médica resaltó la falta de reconocimiento del niño con síndrome de Down y de su madre, como sujetos de derechos, dignos de respeto y significación social.

Los prejuicios frente a la discapacidad y el significado del síndrome de Down que se expresaron en la atención médica reproducen desigualdades sociales y afectan la atención que reciben los niños con síndrome de Down y sus madres; sin embargo, las madres lograron aprendizajes desde su experiencia de crianza que les permitieron transformar esas creencias, reconocer al niño con síndrome de Down como un sujeto, con capacidades y derechos y por tanto resignificar la información que recibieron del médico.

Las madres desde su experiencia reconocieron la necesidad de una atención médica humanizada, la cual se manifiesta con un trato digno y el reconocimiento del sujeto con sus capacidades y necesidades.

\section{Financiamiento y conflicto de intereses}

Se contó con el apoyo del Departamento de Pediatría y Puericultura de la Facultad de Medicina 
de la Universidad de Antioquia y de la Asociación Amigos con Calor Humano, organización social que ofrece servicios de rehabilitación, inclusión social y productiva a personas con discapacidad y sus familias. No hubo conflicto de intereses entre las instituciones vinculadas al proyecto de investigación. El proyecto fue financiado por el grupo de investigación Salud y Sociedad de la Facultad Nacional de Salud Pública de la Universidad de Antioquia.

\section{REFERENCIAS}

1. Toboso Martín M, Arnau Ripollés M S. La discapacidad dentro del enfoque de capacidades y funcionamientos de Amartya Sen. Araucaria. Revista Iberoamericana de Filosofía, Política y Humanidades [en línea] 2008;10:64-94. Fecha de consulta: 7 de marzo de 2017. ISSN 1575-6823.

2. Nussbaum M. Crear Capacidades: Propuesta para el Desarrollo Humano. Iberica P, editor. 2012.

3. Organización Mundial de la Salud; Organización Panamericana de la Salud. Clasificación Internacional del Funcionamiento, de la Discapacidad y de la Salud [Internet]. 2001. Disponible en: http://ccp.ucr.ac.cr/bvp/pdf/desarrollohumano/oms-clasificacion-01.pdf

4. Peña H. Ética y relación médico-paciente en pediatría. Rev. Peru. Pediatr. 2011;64(2):41-4.

5. Mercado FJ. Entre el infierno y la gloria. La experiencia de la enfermedad crónica en un barrio urbano de Guadalajara. Univ Guadalajara. 2002;261-278.

6. Bastidas A M, Ariza Mo G, Zuliani A L. Recognizing a child with Down's syndrome as a subject of parenting. Revista Facultad Nacional de Salud Pública [Internet]. Universidad de Antioquia; 2013;31(1):102-9. Disponible en: http://www. scielo.org.co/scielo.php?script=sci_arttext\&pid=S0120386X2013000100012\&lng=en\&nrm=iso\&tlng=es

7. Huiracocha L, Almeida C, Huiracocha K, Arteaga A, Arteaga J, Barahona P, et al. Explorando los sentimientos de los padres, la familia y la sociedad a las personas con Síndrome de Down : Estudio observacional. Maskana; 2013;4(2):47-57.

8. Molina Saorín J, Nunes Corredeira RM, Vallejo Ruiz M. La percepción social hacia las personas con síndrome de Down : la escala EPSD-1 The social perception of people with Down syndrome : the EPSD-1 scale Jesús Molina Saorín. Educ e Pesqui [Internet]. 2012;38(4):949-64. Disponible en: http://www.scielo.br/pdf/ep/v38n4/11.pdf

9. UNICEF. Estado Mundial de la Infancia 2013: Niños y Niñas con Discapacidad [Internet]. 2013 [cited 2015 May 18]. Disponible en: http://www.unicef.org/ecuador/SPANISH_SOWC2013_Lo_res.pdf

10. Taylor S, Bogdan R. Capítulo 4. Entrevista en profundidad. En: Introducción a los métodos cualitativos de investigación. Barcelona: Paidos, 1994: 100-21.
11. Cisterna F. Categorización y triangulación como procesos de validación del conocimiento en investigación cualitativa. Theoria [Internet]. 2005;14(1):61-71. Disponible en: http:// www.ubiobio.cl/theoria/v/v14/a6.pdf

12. Castillo $E$, Vásquez $M$. El rigor metodológico en la investigación cualitativa. Colomb Med. 2003;34(3):167-167.

13. Gobierno colombiano CDLR. LEY Estatutaria 1618 DEL 27 DE FEBRERO DE 2013.pdf. 2013;(1618):24. Disponible en: http://wsp.presidencia.gov.co/Normativa/Leyes/Documents/2013/LEY 1618 DEL 27 DE FEBRERO DE 2013.pdf

14. Bordieur P, Passeron, Jean Claude. La reproducción: elementos para una teoría del sistema de enseñanza. Ciudad de México: Fontamara, 1996.

15. Lolas F. Bioética y medicina. Santiago de Chile: Editorial Biblioteca Americana; 2002.

16. Bolaña MA. ¿Un nuevo saber social que tome en cuenta la vida cotidiana? La revolución contemporánea del saber y la complejidad social. 2006.

17. Foucault M, Dreyfus H, Rabinow P. Cómo se ejerce el poder. En: Gallimard E, editor. Un Parcours Philosophique. Paris; 1984.

18. Honneth A. Reconocimiento y menosprecio. Sobre la fundamentación normativa de una teoría social. Madrid: Editores K, 2010: 13-40.

19. Menéndez E. Modelo médico hegemónico y atención primaria. Segundas Jornadas de Atención Primaria de la Salud 1988; 30 de abril al 7 de mayo [Internet]. 1988. pp. 451-64. Disponible en: http://www.psi.uba.ar/academica/carrerasdegrado/psicologia/sitios_catedras/electivas/816_rol_psicologo/material/unidad2/obligatoria/modelo_medico.pdf

20. Alarcón M AM, Vidal H A, Rozas JN. Salud intercultural: Elementos para la construcción de sus bases conceptuales. Rev Med Chil. 2003;131(9):1061-5.

21. Foucault M. Los Anormales. Informe del curso de 19741975 dictado por Michel Foucault en el College de France. Buenos Aires: Fondo de Cultura Económica, 2006.

22. Tello F. Las esferas de reconocimiento en la teoría de Axel Honnet. Rev Sociol [Internet]. 2011;26:45-87. Disponible en: http://www.facso.uchile.cl/publicaciones/sociologia/ articulos/26/2603-Tello.pdf

23. Honneth A. La lucha por el reconocimiento. Barcelona: Crítica, 1997: 230.

24. Honneth A. Reconocimiento y obligaciones morales. Rev Int Filos Política. 1996;8(8):5-17.

25. Peñaranda $F$. The individual, social justice and public health. Cien Saude Colet [Internet]. 2015;20(4):987-96. Disponible en: http://www.scielo.br/scielo.php?script=sci_ arttext\&pid=S1413-81232015000400987\&lng=en\&nrm=i so\&tlng=es

26. Ferreira MÁ. Sociología de la discapacidad: investigación y compromiso. Ponencia presentada para el III Congreso Nacional "Universidad y Discapacidad" 2007. 